\title{
Un linguista a cui piaceva il jazz
}

\author{
Massimo Fanfani
}

PUBBLICATO: 10 MAGGIO 2021

S ono ormai tanti gli amici e i colleghi che ci hanno lasciato nell'ultimo anno. E ogni volta si fa più lacerante la tristezza per queste morti disumanamente "distanziate" e prive di compianto, davanti alle quali ci ritroviamo sbigottiti e impotenti. Ma non dobbiamo farci prendere dallo sconforto: chi ci ha preceduto continua a confidarci il suo bene e ciò ci incoraggia a guardare avanti senza timore, ravvivando anche da poche faville la fiamma del ricordo. Una fiamma che dovrà farci luce: adesso e oltre il tempo che verrà.

Il 3I marzo 202I, nell'ospedale di Zemun, municipalità di Belgrado alla confluenza della Sava col Danubio, per l'aggravarsi della malattia provocata dal contagio, anche il linguista Ivan Klajn se n’e andato. Dal 2013 era socio estero dell'Accademia della Crusca. Per la precisione: accademico estero "emerito", e in pensione da più di un decennio. In Serbia, come altrove, gli universitari vanno in pensione a sessantacinque anni, e nonostante avesse avuto la possibilità di tenere ancora un piccolo corso con orario ridotto, Klajn era rimasto assai dispiaciuto quando dové lasciare l'insegnamento attivo.

Al momento della sua elezione alla Crusca non pochi si chiesero chi fosse quello studioso serbo che da tempo viveva appartato e non s'interessava quasi più di lingua italiana. Ma nel corso della sua vita Klajn se n'era occupato approfonditamente, e in modo originale e intelligente, con una selva di contributi di grande valore; aveva svolto, parallelamente a quanto fecero per altri versi Eros Sequi, Nikša Stipčević e Sergio Turconi, una preziosa funzione di raccordo fra la cultura jugoslava e quella italiana; era stato a lungo il principale animatore del Dipartimento di Italianistica della Facoltà di Filologia di Belgrado e direttore di diverse riviste linguistiche, fra cui, fin dalla fondazione nel I975, la gloriosa "Italica Belgradensia", come d'altra parte dirigeva "Jezik danas", l'organo di "Matica Srpska", l'istituzione sorta nel I 826 per la rinascita culturale della nazione serba e lo studio della sua lingua, una specie di "Crusca" slava, modello di numerose istituzioni simili nei paesi dell'Europa centro-orientale.

Klajn era nato il 3I gennaio I937 da una famiglia della buona borghesia del vecchio Regno di Jugoslavia. Il padre, Hugo Klajn (I894-I98I), ebreo originario di Vukovar in Croazia (il cognome "Klein" è tipico di ebrei tedeschi, anche se gli antenati vi eran giunti dalla Polonia), aveva studiato medicina a Vienna. Allo scoppio della Grande guerra dovette interrompere la sua formazione - allora la Croazia apparteneva all'Impero austroungarico - per arruolarsi come medico di battaglione in Galizia e in Italia. Dopo aver seguito un corso di psicanalisi, assistette alle conferenze di Freud alla Berggasse, divenendo, negli anni venti, il primo psicanalista a esercitare nel Regno di Jugoslavia e lavorando nell'ospedale psichiatrico di Guberevac a Belgrado. Nel 1937 aveva sposato Stana Đurić (I905-1986), già moglie di Vladislav Ribnikar, proprietario del quotidiano "Politika", e madre di tre bambini. Stana aveva trascorso la sua fanciullezza in Francia, dove la famiglia si era rifugiata per la guerra, e si era perfezionata in pianoforte a Parigi col celebre virtuoso e compositore Lazare Lévy, iniziando giovanissima un'intensa attività concertistica e fondando nel 1932 a Belgrado la rivista musicale "Zvuk".

Quando giusto ottant'anni fa, nell'aprile 194I, Hitler e Mussolini invasero il Regno di Jugoslavia bombardando massicciamente Belgrado e scatenando un lungo e sanguinoso conflitto di tutti contro 
tutti, conflitto che costò alla popolazione un milione di morti e si accompagnò, fra le altre nefandezze, a una delle più feroci persecuzioni degli ebrei, i Klajn persero tutto e riuscirono a salvarsi fortunosamente solo grazie all'interessamento del nonno materno di Ivan, Mihailo Đurić, stimato presidente della Camera di Commercio. Hugo Klajn dovette comunque cambiare identità e separarsi dalla famiglia per tutto il tempo della guerra, facendo recapitare al piccolo Ivan delle cartoline di Venezia, così da fargli credere che si trovasse là.

Questa drammatica esperienza segnò profondamente i Klajn. Alla fine della guerra, la bella casa nel centro di Belgrado di fonte alla fortezza Kalemegdan incendiata e ridotta in macerie, quasi tutta la famiglia paterna scomparsa nel famigerato campo di Jasenovac, bisognò ritrovare il coraggio per ricominciare da capo. Il padre, dopo aver scritto nel 1945 il suo ultimo saggio scientifico dedicato alle nevrosi di guerra, col nuovo regime comunista fu nominato direttore del Teatro Nazionale di Belgrado e si converti all'attività teatrale: regista, critico, traduttore, insegnante all'Accademia di cinema e teatro, elaborò un particolare metodo di recitazione denominato "sistema KlajnStanislavski". La madre non tenne più concerti, ma si dedicò agli studi musicologici e all'insegnamento: fra i suoi libri una storia della musica serba. Ivan imparò presto a suonar pianoforte, ebbe un'eccellente formazione letteraria e, come spesso avviene nei Balcani - basti ricordare il poliglottico caleidoscopio dell'infanzia a Rustschuk descritto da Elias Canetti - fu indotto fin da piccolo a conoscere piu lingue. E infatti possedeva bene, oltre alle lingue classiche e alle principali lingue romanze, l'inglese, il tedesco, il russo.

La madre parlava fluentemente francese; in casa si usava il tedesco, la lingua che il padre aveva affinato a Vienna; l'inglese venne da sé, insieme col jazz. Va ricordato che dopo la rottura di Tito dalla Russia di Stalin e l'uscita dal Cominform nel I948, la Jugoslavia, desiderosa di rimarcare la sua autonomia, si era ben presto aperta alla cultura occidentale, tanto che la musica che allora andava per la maggiore a Belgrado era quella di Benny Goodman e Glen Miller. Klajn, che fin da ragazzo era dotato di talento e aveva studiato armonia e contrappunto con la compositrice Ljubica Marić, attraverso la madre, libera di recarsi all'estero, era riuscito a procurarsi non pochi spartiti di musica jazz e dei musical di Broadway. Così, suonando per gli amici, l'inglese cominciò a mescolarsi alle note. L'interesse per l'italiano nacque invece dall'amore per la bellezza della poesia.

Al momento d'iscriversi all'università, consapevole di conoscer già diverse lingue, Klajn, seguendo le orme del fratello maggiore, scelse Chimica fisica, colla speranza di trovar facilmente lavoro. Ma la passione per le lingue non era un capriccio di gioventù, ma una vocazione profonda: così l'anno seguente cambiò facoltà. In quegli anni a Belgrado, come del resto a Zagabria, erano attivi diversi ottimi linguisti e storici dell'italiano, in particolare Momćilo Savić, Srđan Musić e Sergij Šlenc, i quali, fra l'altro, nel 1966 avrebbero curato insieme una buona raccolta commentata di testi italiani antichi. Musić si occupava soprattutto di interferenze linguistiche: nel I972 pubblicherà a Belgrado un volume sui prestiti d'origine romanza nella regione delle Bocche di Cattaro e saggi come La quantità vocalica negli italianismi delle Bocche di Cattaro ("Italica Belgradensia", I975); Savić, che sarebbe diventato il maestro di Klajn, prediligeva invece le questioni morfologiche e sintattiche: si deve a lui la prima messa a fuoco della categoria del futuro anteriore nel passato col volume Temporalni kondicional italijanskom jeziku (Belgrado, I966) e cogli articoli, Il condizionale temporale nelle tre redazioni del romanzo manzoniano (in "Linguistica", I963); Il trapassato remoto nelle tre redazioni del romanzo manzoniano (ivi, 1972); interessante anche un suo scritto del 1964 sul problema della periodizzazione, su fondamenti interni, della storia linguistica italiana.

Klajn dopo aver discusso nel 1964 una tesi magistrale sulle parole di origine straniera nellitaliano del dopoguerra, si addottorò nel I970, sotto la guida di Savić, col lavoro Influssi inglesi nella lingua italiana 
che, in forma ridotta, sarebbe diventato il noto volume pubblicato nel I972, a Firenze, negli "Studi" dell'Accademia Colombaria. Si tratta di un'opera straordinaria, non solo perché costituisce la prima e finora insuperata descrizione complessiva dei fenomeni d'interferenza inglese, ma soprattutto per l'originale impianto teorico, fondato su assunti nuovi e una concezione aperta e coraggiosa: «la "relatività" di tutti i prestiti e soprattutto di quelli non lessicali: lo "straniero", cioè, non è un elemento alieno, da contrapporre all'"indigeno" o "ereditario", ma una forza catalizzatrice che agisce solo se in armonia con le tendenze intrinseche del sistema ricevente. Per questo [...] si è spesso cercato, piuttosto che dimostrare l'influsso inglese, di mettere in luce le cause interne, finora inosservate, di un presunto o parziale anglicismo».

Frattanto, fin dagli anni dell'università, aveva cominciato a lavorare come giornalista e traduttore: un'attività mai intermessa, necessaria per arrotondare il non lauto stipendio e talvolta per sopravvivere, ma anche fonte di idee e stimoli per la riflessione linguistica. Klajn ha tradotto, per lo più dall'italiano e dall'inglese, opere letterarie (nel I992 un suo adattamento del Candelaio di Giordano Bruno venne messo in scena a Belgrado), scientifiche e perfino fumetti: fra questi gli albi francesi di "Asterix" e quelli belgi di "Tintin", ai cui paronimi o malapropismi nel 2005 dedicò un saggio. Come giornalista collaborò a vari quotidiani e settimanali, a lungo incaricato della redazione di articoli per le pagine degli esteri, ciò che gli consentiva di passare in rassegna giornali e riviste straniere.

Nel 1974 gli fu affidata per la prima volta una rubrica linguistica su "Borba", il principale quotidiano jugoslavo, organo della Lega Comunista, e da quel momento i suoi interventi per il largo pubblico, in ben indovinate rubriche linguistiche riprese da un giornale all'altro, si sono infittiti, riscuotendo un successo crescente. Da più di un ventennio teneva una rubrica sulla rivista "Nin", puntuale, ogni settimana, come un esperto giornalista che sa il suo mestiere: l'ultimo pezzo, sulle novità ortografiche della scrittura digitale, è apparso lo scorso 25 marzo, quand'era già da qualche giorno in ospedale. Dai suoi interventi giornalistici aveva potuto ricavare via via diversi fortunati volumi divulgativi. In una rivista aveva collaborato anche alla sezione enigmistica: una scelta di anagrammi, palindromi, pangrammi e altri giochi di parole che vi aveva escogitato confluirono nel volume I filozofi su ludi. Antologija smešnih štamparskih grešaka ["E i filosofi sono pazzi. Un'antologia di divertenti errori di stampa"] del 2007 .

Parallelamente all'attività giornalistica proseguiva la sua formazione e il suo lavoro di linguista. Già nei primi anni sessanta erano apparsi alcuni suoi articoli sul problema della traduzione del congiuntivo in serbo-croato, sulla pronuncia e le condizioni per l'adattamento delle parole straniere in italiano. Per le sue ricerche era venuto in Italia e dal 1968 al 1970 aveva avuto una borsa come lettore di serbo-croato all'Università di Firenze, dove lo aveva accompagnato la moglie Aleksandra, che aveva studiato lingue anchessa e provvedeva al loro sostentamento lavorando come commessa in una bottega del Ponte Vecchio. A Firenze Klajn conobbe Devoto e Nencioni, e strinse amicizia con Cosimo Pekelis e Anton Maria Raffo. Era stato Migliorini a pubblicare nel I967, in "Lingua nostra", il suo primo saggio in italiano col quale s'inquadravano in una nuova prospettiva i nessi consonantici. E alla medesima rivista Klajn destinò poi una serie di contributi volti quasi tutti a chiarire, in modo esemplare e convincente, diversi nodi grammaticali: le funzioni del pronome esso (I976), l'uso del pronome riflessivo tonico (I979), i dimostrativi e la deissi (I986), il ci impersonale (I99I).

Il biennio trascorso a Firenze, se consenti a Klajn di portare a compimento il lavoro di dottorato (predisponendone la versione italiana che, come s’è detto, sarà pubblicata nel 1972) e di andare alla scoperta dei tesori artistici della città, per il resto fu per lui piuttosto penoso. Infatti, nonostante la buona volontà, non riusci a tenere che poche lezioni, tanto che quella prima esperienza d'insegnante di serbo-croato fu un vero fallimento. Proprio in quel biennio r968-rg69 (e sovente anche dopo, fino ai 
primi anni novanta) la nuova e moderna sede della Facoltà di Lettere in piazza Brunelleschi fu occupata da assemblee studentesche e da bivacchi di giovani rampolli della Firenze bene, che di fatto impedivano lo svolgimento delle lezioni e il normale uso delle sale di studio. In tale situazione, coloro che avrebbero dovuto, come maestri, assumere comportamenti responsabili e pronunciare parole assennate e veritiere, o si rinserrarono nei loro eburnei torrioni, o cercarono di cavalcare la tigre, proclamando la morte della vecchia università e l'apertura ai giovani, mentre si trastullavano magari con le teorie linguistiche di Stalin: cosi facendo avviarono quella che era una facoltà non mediocre verso una china rovinosa e senza fine. Andò meglio per Klajn quando ottenne una borsa Fulbright per gli Stati Uniti e nel 1983-1984 fu lettore a Yale nel Connecticut. Con pochi studenti, suonando canzoni del cantautore Đorde Balašević, così da abituare il loro orecchio alla melodia della lingua slava con le sue vocali di diversa lunghezza e le quattro tonalità dei suoi accenti, riuscì a insegnare in modo davvero efficace e per lui di piena soddisfazione.

Oltre al volume sugli anglicismi, Klajn veniva pubblicando in riviste jugoslave numerosi altri interventi sullitaliano, dedicati soprattutto alla morfologia o ad analisi di tipo contrastivo. Fra gli articoli scritti in italiano si segnalano: La definizione della parola composta e $i$ composti in italiano ("Živi jezici",I972); Intorno alla classificazione delle parole composte (ivi, 1974); Intorno alla definizione del pronome ("Linguistica", I975); Funzioni dei pronomi personali in italiano ("Italica Belgradensia", I989); Pronomi, avverbi e preposizioni (in "Linguistica", I99I); Subordinate esplicite introdotte da preposizioni ("Italica Belgradensia", I995); Sul trattamento lessicografico della particella "ne" (ivi, 2004).

Anche alla lingua della nostra tradizione poetica, che fino ad allora non era stata oggetto d'indagini specifiche, Klajn riservò una serie di finissimi lavori: Carducci e il linguaggio poetico tradizionale ("Linguistica", I972), Per una definizione del linguaggio aulico della poesia italiana ("Italica Belgradensia", 1975), Pascoli e la fine del linguaggio aulico ("Filološki pregled", 1979).

Frattanto teneva corsi all'Università di Belgrado, e non solo di Lingua italiana. Per un certo periodo insegnò Grammatica storica dello spagnolo (e ne compilò un manuale nel i977); poi Linguistica applicata e Grammatica comparata delle lingue romanze. Nel 1984 sarà nominato professore ordinario, coprendo successivamente, per due volte, la carica di direttore di dipartimento. Corrispondente dell'Accademia Serba delle Scienze e delle Arti, nel 2003, l'anno successivo al pensionamento, ne era divenuto socio ordinario.

Verso la metà degli anni novanta si era rivolto all'allora presidente della Crusca, Giovanni Nencioni, sperando di riuscire a far stampare in Italia un volume con una scelta dei suoi scritti italianistici, visto lo scarso riscontro che avevano trovato da noi. Il progetto, a cui teneva molto, sfumò miseramente e la cosa lo amareggiò. Negli anni seguenti portò a termine un grande vocabolario italo-serbo di quasi cinquantamila lemmi (Italijansko-srpski rečnik, Belgrado, 1996), ristampato poi in sei nuove edizioni: un'impresa lessicografica notevole, compiuta "da un solitario laboriosissimo operaio", come scrisse Raffo: "la fraseologia è ricca e aggiornata, mentre un'altra utilissima novità è costituita dalla separazione e classificazione dei significati. [...]. Inoltre, di grande profitto per l'utente serbo o croato l'appendice, recante un'accurata sintesi della grammatica italiana, nonché un elenco dei verbi irregolari e difettivi, dove, anziché lacune, trovo semmai qualche preziosa ridondanza". Nel 2000 pubblicò ancora un manuale per l'università, Esercizi di lessicologia e fraseologia italiana; tuttavia i suoi interventi sul fronte della linguistica dell'italiano andarono progressivamente diradandosi, anche perché era divenuto estremamente difficoltoso continuare a studiare in modo serio a Belgrado: le biblioteche non acquistavano nemmeno le riviste e i libri più importanti, da parte italiana non giungeva alcun sostegno, mentre i viaggi di studio all'estero si facevano sempre più radi. Erano quelli gli anni in cui la Jugoslavia stava precipitando verso la sua dissoluzione attraverso una sequela di guerre sanguinose 
culminate, nel I999, con l'aggressione alla Serbia e lo sconsiderato bombardamento di Belgrado, vicende nelle quali anche l'Italia ebbe la sua parte.

Fra le tante conseguenze di quei nuovi tragici conflitti ci fu l'innaturale divisione del serbo-croato, lingua parlata con minime differenze nelle varietà locali di Serbia, Croazia, Bosnia-Erzegovina e Montenegro, come se si trattasse di lingue distinte. Una divisione dovuta solo a motivi politicoideologici, ma che ha prodotto disorientamento, ha aumentato le incomprensioni, ha portato a nuove incongruenze, come, ad esempio, la pratica di ritradurre lo stesso testo nei diversi paesi, ma in lingue che si assomigliano più o meno come si assomigliano le varietà regionali dell'italiano. A questa artificiale divisione del serbo-croato Klajn, da buon linguista, ha sempre guardato con scetticismo. E nel 2017 è stato fra i firmatari dell'appello di Sarajevo a favore della lingua comune.

Oltre all'italiano, anche la sua lingua materna lo aveva appassionato, destando il suo interesse di studioso, e non solo per via delle rubriche linguistiche che teneva nei giornali. Fra i diversi lavori scientifici dedicati al serbo-croato, vanno ricordati almeno lo studio sulle funzioni e la natura dei pronomi (O funkciji i prirodi zamenica, Belgrado, 1985) e i due volumi sulla morfologia derivativa del serbo (Tuorba reči u savremenom srpskom jeziku, Belgrado-Novi Sad, 2002 e 2003). Ma nel 1997, con la costituzione del Consiglio per la standardizzazione della lingua serba, nato in conseguenza della separazione linguistica cui si è accennato, anch'egli, come uno dei principali linguisti attivi a Belgrado, si trovò incaricato di provvedere al complesso processo di nuova codificazione del serbo, un compito che ha assolto coscienziosamente, con una visione libera e aperta della lingua, sobbarcandosi un'enorme mole di lavoro ma realizzando comunque alcune pregevoli opere di riferimento che hanno consentito ai suoi concittadini di conoscere piu a fondo la loro lingua nei suoi rapporti con le lingue di altre popolazioni: un repertorio di dubbi linguistici (Rečnik jezičkih nedoumica, apparso già nel I98I, ma sempre aggiornato e giunto ora alla tredicesima edizione); una grammatica (Gramatika srpskog jezika, Belgrado, 2005; nel 2007 ne è uscita una anche in lingua italiana) e, insieme a Milan Šipka, un grande dizionario di termini e locuzioni forestiere del serbo (Veliki rečnik stranih reči i izraza, Novi Sad, 2006).

Da molto tempo, ormai, non viaggiava più allestero. Ma quando ancora veniva a Firenze, scendeva in un piccolo albergo vicino alla stazione di rimpetto a un grande magazzino dove la moglie Saša, come capitava ad altri visitatori d'oltre cortina, passava ore a riempirsi gli occhi di quel che non avrebbe mai visto a Belgrado. Ivan, invece, con passo lento e legnoso, l'alta figura avvolta in un nero soprabito, il volto scarno incorniciato da un'insolita barba di foggia ottocentesca, serio e assorto lo sguardo dietro le lucide lenti, faceva il giro delle biblioteche alla ricerca di libri che non avrebbe trovato in patria. Laconico ma rapido nel cogliere ogni possibile spunto di dialogo, severo nei giudizi ma dotato di arguzia e humor sottile, estremamente riservato ma pieno di attenzioni per l'interlocutore, nonostante l'innata modestia e la barriera della sua ritrosia, si avvertiva il fuoco del suo ingegno, la sterminata cultura, la profonda umanità. Le domande che poneva davano sempre da riflettere e incutevano rispetto. Forse proprio per queste non comuni qualità Klajn non aveva molti amici, né in patria né fuori.

Dopo la scomparsa della moglie, avvenuta qualche anno fa, aveva chiuso il pianoforte. E col progredire dei disturbi alla vista si era affidato alla penombra del suo studio nella modesta abitazione di Zemun, dov'era sempre vissuto da quando si era sposato. Continuando a sbrogliare con la mente i garbugli della lingua, ancora scrivendo i pezzi che i giornali gli richiedevano, ricevendo le visite dei suoi antichi scolari, come Mila Samardžić e Julijana Vučo, che mai l'avevano dimenticato: "Ogni materia che insegnava - ed era impressione diffusa che le potesse insegnare tutte - era una fonte inesauribile di informazioni e particolari individuati solo da lui, che nessun altro suo collega sapeva 
rilevare con tanta sensibilità e poi tramandarli in maniera estremamente divertente e brillante: gli esempi con i quali egli illustrava la lingua italiana, dalle strutture ai modi di dire, saranno ricordati da numerose generazioni come veri e propri modelli di insegnamento linguistico". Perché è vero che Klajn era un uomo austero e aveva pochi amici, ma a conoscerlo solo un po', come avevano potuto conoscerlo e ammirarlo i suoi tanti scolari, non si poteva non volergli bene.

\section{Cita come:}

Massimo Fanfani, Un linguista a cui piaceva il jazz, "Italiano digitale", XVII, 2021/2 (aprile-giugno) DOI: 10.35948/2532-9006/2021.8541 